\title{
Trois pièces à sujets antiques de Marguerite Yourcenar
}

\author{
FRANÇOIS JOUAN \\ Université de Paris $X$, Nanterre
}

\begin{abstract}
RESUMO: Marguerite Yourcenar inspirou-se, em suas obras, tanto na Grécia antiga quanto na Grécia moderna. De dois volumes do seu teatro completo, o segundo é consagrado a três "peças gregas": Electra ou a queda das máscaras, O mistério de Alceste e Quem não tem seu minotauro? - seguidas de estudos para cada uma das peças, admiráveis páginas de crítica e de estilo. Caracterizam essas obras questőes metafísicas que permeiam a sua produção poética ou romanesca e que giram em redor do Homem, da Liberdade e da Fatalidade, da Personalidade e do Inconsciente. Apesar da inteligência, da cultura, da originalidade e da beleza do estilo, estas peças não têm qualidades propriamente dramáticas, daí a raridade das suas representaçöes.

PALAVRAS-CHAVE: Marguerite Yourcenar, teatro grego antigo, teatro moderno.
\end{abstract}

On sait la place considérable que tient dans l'oeuvre de Marguerite Yourcenar l'inspiration grecque, nourrie par un contact direct avec les oeuvres au cours des années et par une bonne pratique de la Grèce moderne ${ }^{1}$. Pour la Grèce des origines et des mythes, il suffit d'évoquer son recueil poétique de Feux et son théâtre; pour la poésie antique son Pindare et ses belles traductions de La Couronne et la Lyre; pour la Grèce au temps de l'Empire Romain, les Mémoires d'Hadrien, son oeuvre la plus célèbre; pour la Grèce byzantine et moderne, certaines de ses Nouvelles Orientales et sa belle étude de la poésie de Cavafy. Mais cette liste est loin d'être limitative: la Grèce est partout dans son oeuvre.

Des deux volumes de son théâtre complet, le second est consacré à ses trois "pièces grecques": Electre ou la Chute des Masques, écrite en 1944, Le Mystère d'Alceste, de 1943, et Qui n'a pas son Minotaure?, achevée sous sa forme définitive en 1957. Au texte des pièces s'ajoute dans l'édition Gallimard de 1971 une étude sur chacun de ces drames (soit une cinquantaine de pages au total), où l'auteur a indiqué ses sources et précisé ses intentions. Très précieuses, naturellement, pour interpréter les oeuvres, ces pages de critique sont remarquables par la richesse et la sûreté de l'information, la justesse et la profondeur des idées et le bonheur du style. Plusieurs critiques ont rangé ces morceaux parmi les meilleurs écrits de Marguerite Yourcenar, un avis que pour ma part j'approuve entièrement. 
Dans le bref temps dont je dispose, je ne pourrai que donner une idée générale de ces trois pièces, espérant vous inviter ainsi à les lire ou les relire, en insistant seulement sur quelques scènes dont l'écrivain a signalé expressément l'importance à ses yeux.

Marguerite Yourcenar a dit quelque part que le mythe grec était un "chèque en blanc" offert à l'écrivain moderne. La manière dont elle-même a rempli ces blancs combine la fidélité à l'esprit du mythe avec une grande liberté dans son traitement. Ainsi, avec Electre ou la Chute des Masques, un des mythes les plus connus de l'antiquité se trouve renouvelé par la modification des caractères et le transfert des situations. Pour l'essentiel, la dramaturgie est celle de l'Electre d'Euripide, même si l'auteur ne s'interdit pas les emprunts à Sophocle ou à Eschyle. Mais dès la première partie, qui décrit les préparatifs de vengeance des héritiers d'Agamemnon, des failles imprévues apparaissent dans les caractères: si Pylade est jaloux de Théodore - le faux époux d'Electre - celle-ci n'est pas moins jalouse de l'affection un peu trop étroite qui unit les deux amis. Quant à Oreste, il se dit trop faible pour tirer l'épée contre sa mère, mais il tuera sans hésitation Egisthe, quoiqu'il garde de cet hôte de sa famille de meilleurs souvenirs d'enfant que de son propre père.

Mais c'est dans la seconde partie. de la pièce, celle de l'exécution de la vengeance que les masques, déjà ébranlés, vont tomber: devant Electre, Clytemnestre justifie sa grande passion pour Egisthe en rappelant sa situation de jeune femme mariée à un soudard, abruti par des années de campagnes coloniales, ambitieux, affairiste et coureur: Agamemnon. Quant à Electre, déjà jalouse d'une mère qui a pleinement réussi sa vie de femme, lorsqu'elle est raillée par celle-ci pour avoir été en secret amoureuse d'Egisthe, elle est saisie d'une rage folle, se précipite sur sa mère et l'étrangle: il n'y a que la vérité qui blesse!

Un peu plus tard, l'arrivée d'Egisthe va déjouer tous les projets des conjurés: il semble heureux de découvrir auprès d'Electre Pylade et Oreste. Il accueille avec flegme la nouvelle de la mort de Clytemnestre, car atteinte d'un mal incurable elle s'est vue ainsi, dit-il, épargner quelques mois de souffrances. On découvre ensuite que pendant toutes ces années d'exil, Egisthe avait soudoyé Pylade pour entretenir Oreste et avoir de ses nouvelles. Celui-ci s'aperçoit alors avec consternation qu'il n'a été qu'un jouet entre les mains de sa soeur et de son ami. Ultime révélation: Oreste est en réalité le fils d'Egisthe, et si le couple a tué Agamemnon, c'était pour l'empêcher de découvrir la vérité. Ainsi Clytemnestre aimait cet enfant de l'amour, Egisthe veillait sur lui de loin et il est tout prêt à lui rendre le trône de Mycènes. Que fera Oreste? Incapable de s'adapter à une situation si nouvelle, contre toute logique il poingnardera son père, et celui-ci, par une ultime générosité, favorisera la fuite de ses trois meurtriers. Le trio s'exilera, lié à jamais par ce crime exécuté en commun, voué à une sorte de huis-clos sartrien d'amour et de haine. Et c'est l'innocent Théodore, survenu mal à propos, qui paiera pour les deux meurtres. 
Dans l'Examen d'Alceste, Marguerite Yourcenar considère que de ses trois pièces antiques, celle-ci est la plus proche du modèle grec. Son dessein a été "de rénover pieusement une légende antique pour la rendre, s'il se peut, plus immédiatement accessible." Ici encore, on retrouve l'essentiel des scènes du drame d'Euripide, même si les séquences en sont parfois modifiées. Mais par toute une série de "coups de pouce", l'auteur a profondément altéré la forme et même l'esprit du récit euripidéen. En effet, hostile à toute "reconstitution archéologique", elle a cherché, dit-elle, à renforcer le caractère intemporel donc éternel, de ce récit "de sacrifice et d'héroisme". Ainsi, Admète n'est-il plus un roi mais un proprétaire aisé. Alceste s'est sacrifiée pour lui à son insu, Admète étant victime "d'une vieille malédiction familiale", et sa mort offrira l'apparence d'une longue et mystérieuse maladie. Thanatos n'est plus un génie ailé masculin, mais prend la figure plus familière à un public moderne d'une vieille femme enveloppée de voiles noirs. Il n'y a pas de choeur: il est remplacé par une vieille servante, Georgine, et ponctuellement par une troupe de cinq voisines qui viennent jacasser devant la porte de la mourante. Mais les changements les plus notables se situent à l'intérieur du couple. Malgré leurs marques d'affection mutuelle et le réel désespoir d'Admète, le dévouement d'Alceste est loin d'être serein. Elle se plaint de n'avoir pas été comprise par son mari: "Tu vivais, dit-elle, dans un palais dont $\mathrm{j}$ 'habitais au plus le jardin". Admète, de son côté, dénonce le manque d'intérêt de sa femme pour ses talents de poète et de musicien, sa sensualité, sa jalousie. Cette Alceste qui s'en va est partagée entre l'amour et la rancune, sinon la haine, et sa mort a des allures de suicide.

A peine Alceste est-elle morte qu'arrive le cortège des importuns; l'Entrepreneur des Pompes funèbres; à la place du Phérès d'Euripide, les parents d'Admète, deux vieillards égoistes, avares, radoteurs, qui n'avaient jamais accepté leur belle-fille; puis le Maire du village. Dernier importun: une sorte de vagabond, sale, débraillé, malodorant: Hercule. Le premier réflexe de l'hospitalier Admète, excédé, est de le chasser. "Dehors, qu'on le foute à la porte!". Il ne se ravise que quand Georgine lui rappelle que "Madame recevait toujours bien les mendiants." Il le fait donc conduire au pavillon des hôtes.

C'est au cours de la veillée funèbre, confiée aux femmes, que surgit Hercule ivre, pourchassant la petite servante Phyllis. Il apprend alors qui est la défunte. Subitement dégrisé et honteux, il décide de se racheter en arrachant Alceste à la mort. Ce combat d'Hercule avec la mort est considéré par l'auteur comme le centre du drame, ce qu'elle a appelé la "scène à faire". Ce n'est pas une lutte physique, comme celle qu'Euripide rejette du reste en coulisses, mais un affrontement moral, un choc de volontés dans les ténèbres, un duel verbal. La Mort prend sucessivement toutes les apparences, use de tous les tons et de tous les arguments pour triompher d'Hercule et emmener Alceste. Le héros, un simple homme bien que fils de Zeus, fait front de son côté dans un combat inégal, avec sa santé d'athlète, son bon sens, sa sincérité, son sens de l'honneur, armes imparables. Avec rage, la Mort lâche prise, mais elle l'avertit que la partie n'est qu'à moitié gagnée. Il devra encore faire accepter à Alceste le retour à la vie. De fait, ni l'évocation de son mari, ni celle de ses enfants, encore moins celle de sa vie passée ne peuvent l'arracher à cette espèce d'insensibilité minérale qui la retient. Pour y parvenir, 
il faut la bonne grosse humanité d'Hercule, son soufle, sa chaleur, l'odeur de son vieux manteau. Alceste se donnerait même à Hercule si celui-ci ne se récusait, avec une délicatesse inattendue de sa part.

Après son retour auprès d'Admète, la pièce se termine en mineur, dans une atmosphère un peu onirique, loin des "grands mots". Alceste déclare: "J'avais sommeil, je m'étais assoupie dans le jardin... Je m'étais égarée... Ce brave homme m'a ramenée. C'est une espèce de garde-champêtre." Et la vie retrouve son cours, tandis qu'Hercule reprend la route...

A la difference des deux premières pièces, Qui n'a pas son Minotaure? a été remanié par l'auteur à plusieurs reprises, à partir d'une première esquisse en 1932, qui était un peu un jeu, jusqu'à sa forme et à son titre définitifs en 1957. C'est dire qu'elle a particulièrement tenu au coeur de Marguerite Yourcenar, et nous sentons qu'elle y a mis beaucoup d'elle-même. Ici, pas de modèle tragique, puisque les pièces de Sophocle et d'Euripide sur le sujet ont disparu. C'est de la légende même, racontée par divers auteurs et illustrée par une riche iconographie antique, que l'écrivain a tiré la matière de ce qu'elle a appelé un "divertissement sacré". Le temps que couvre l'action va de l'arrivée du navire portant Thésée et les ôtages en vue des côtes de Crète à son retour en rade du Pirée, et la structure dramatique comporte dix scènes sucessives: trois sur le navire (la vigie en haut du mât; les ôtages dans la cale; Thésée sur le pont); trois en Crète (le débarquement et la rencontre de Thésée avec Phèdre et Ariane, puis Minos; l'arrivée du prince à la porte du Labyrinthe; enfin dans un "terrain vague au bord de la mer" où se prépare, après la sortie du Labyrinthe, la fuite de Thésée et des deux filles de Minos); deux scènes sur une plage de Naxos, avant et après la séparation de Thésée et d'Ariane; une demière scène enfin avant le débarquement triomphal au Pirée de Thésée et de Phèdre, avec l'épisode de la voile noire, la mort d'Egée et l'avènement du prince héritier.

Mais là encore les traditions antiques sur cet exploit de Thésée (elles-mêmes riches de variantes) sont profondément modifiées, qu'il s'agisse du caractère des protagonistes, de la marche des événements ou du sens du mythe.

Le personnage le moins conforme à son modèle héroique est assurément celui de Thésée, dont on peut dire qu'il devient ici un anti-héros. Il reconnait lui-même que son passé se réduit à "de minces exploits de légionnaire aux colonies" et à "des amours de garnison ou de villes conquises". Arrivé en vue de la Crète, il ne sait même pas ce qu'il va faire, combattre le Minotaure ou plus platement accomplir sa mission de remettre à Minos les victimes que lui envoie Egée en vertu d'un traité, voire - tentation fugitive, à vrai dire - se joindre aux ôtages. Sur le rivage même, la jeune Phèdre se jette à son cou et dès le soir elle se donnera à lui, mais elle le droguera dans la cabine du bateau et fera enlever les ôtages, aussitôt livrés au Minotaure. Ayant manqué, comme il dit 'l'heure de l'embarquement pour la gloire", il arrive trop tard devant la porte fermée du Labyrinthe. Apparaît alors Ariane qui, par amour, veut faire de lui un vrai héros et lui 
entr'ouvre la porte. Il "se résigne à l'héroisme" et pénètre dans le domaine du monstre, relié à la jeune fille par le fameux fil. Nous reviendrons d'ici peu sur cette scène capitale du Labyrinthe. Disons seulement que lorsque Thesée en sort, le Labyrinthe s'effondrant sur lui comme un château de cartes, et qu'il retrouve les deux soeurs sur le rivage, il n'a guère augmenté son capital de gloire.

Les deux filles de Minos, la. blonde Ariane vêtue de blanc et la brune Phèdre, vêtue de rouge, s'opposent dans la pièce comme les deux représentations de l'amour éthéré et de l'amour sensuel, de l'Aphrodite Ourania et de la Pandémos. Phèdre s'était aussitôt donnée à Thésée et s'était moquée de lui. Ariane lui promet d'être son épouse pour la vie et lui fournit l'occasion d'accomplir un bel exploit. Contre l'avis de Thésée, elle insiste pour emmener sa soeur avec eux. Mais le vaisseau retenu par les calmes à Naxos, elle s'apercevra vite qu'avec tout son amour elle n'a pu changer la nature de Thésée. Lui-même récuse l'image d'un Thèsée parfait, il s'ennuie d'Athènes et de la politique et trompe Ariane avec Phèdre. C'est finalement Ariane elle-même, cruellement déçue, qui va renvoyer à Athènes ce médiocre héros avec sa frivole soeur, afin de reprendre sa recherche personnelle de la perfection, dans une solitude qui ne sera troublée que par l'intrusion de Bacchus-Dieu.

Aux personnages du mythe (Minos ne fait qu'une courte apparition, en chef d'État pompeux et ridicule), Marguerite Yourcenar a ajouté un serviteur de Thésée, un marin qu'elle a nommé Autolycos (nom du grand-père maternel peu recommandable d'Ulysse, héros de la comédie et du drame satyrique grec), moitié valet de comédie, comme Sosie, moitié petit dieu malicieux. Il prononce le prologue d'exposition, juché en haut du mât, puis dialogue avec Thésée sur le pont. A terre, il l'assiste en lui prodiguant de bons conseils que son maître ne suit pas. A l'arrivée à Athènes, il endosse philosophiquement la responsabilité d'avoir laissé la voile noire, mais en profite pour se faire donner par Thésée le navire que celui-ci abandonne pour se consacrer à ses réformes politiques.

Marguerite Yourcenar a dit que trois scènes de cette pièce "comptaient parmi celles qui'il lui importait le plus d'avoir écrites". La première est la scène où les quatorze victimes vouées au Minotaure, enfermées dans la cale obscure et ballottées par la houle, réagissent, chacune à sa manière, à une mort toute proche: panique, révolte, résignation, acceptation pieuse ou mystique, détachement, refus de penser. Il suffira de rappeler à ce propos que la pièce a été écrite aux Etats-Unis, dans les dernières années de la guerre, et que bien des consciences, comme celle de l'auteur, étaient bouleversées par les atrocités de l'Europe occupée, dont les massacres d'ôtages.

La seconde scène est celle du Labyrinthe. Thésée avance, seul dans l'obscurité, au milieu d'un concert de voix discordantes: ce sont en fait celle de Thésée enfant, celle de Thésée jeune homme révolté contre son vieux père, mais aussi celles des témoins de sa vie: Autolycos, son ami Lachès, les femmes qu'il a aimées, l'Amazone Antiope et la "petite Hélène", celles qu'il désire, Ariane et Phèdre. Il entend ensuite les voix du Thésée vieilli qui condamnera son fils à mort et croira encore à l'innocence de Phèdre, mais il n'y comprend rien, conclut qu'il s'agit d'Egée et frappe dans le vide en croyant 
frapper son père. Il n'a pas su reconnaître que son adversaire, c'était lui-même, il n'a pas compris que le Minotaure n'existe pas, car chacun secrète son propre monstre, d'où le titre de la pièce.

Je passerai plus vite sur la troisième scène qui est celle de la recontre d'Ariane et de Bacchus, qui, dans la dernière version, devient simplement "Dieu". La fille de Minos commence par diriger ses sarcasmes contre le dieu de la mythologie, mais peu à peu celui-ci prend la stature d'un dieu universel, qui va jusqu'à englober en lui-même le Minotaure. Ariane, fascinée, finit par s'abandonner dans son sein et est entraînée par une sorte de tourbillon ascendant vers une apothéose où elle se fondra parmi les éléments de l'univers, dans l'éternité astrale de la "couronne d'Ariane".

On retrouve donc dans le théâtre antique de Marguerite Yourcenar les grandes questions métaphysiques qui sont au coeur de ses oeuvres poétiques ou romanesques et qui tournent autour de l'Homme, de la Liberté et de la Fatalité, de la Personnalité et de l'Inconscient. On trouve encore le postulat yourcenarien de la supériorité de la femme sur l'homme (avec Clytemnestre, Electre, Alceste, Ariane), les tourments affectifs du trio (deux hommes et une femme dans Electre; deux femmes et un homme dans le Minotaure). La fragilité du couple et le rejet d'un monde de mensonge et de corruption, qui ne laisse que le choix de la mort (Alceste) ou de la solitude (Ariane). Citons encore le penchant vers une vision onirique du monde (la part du lyrisme et du rêve étant particulièrement forte dans le Minotaure).

Divers procédés stylistiques contribuent pour leur part à la "modernisation" du mythe: large intégration des thèmes judéo-chrétiens aux motifs antiques; allusions à l'actualité et usage plus ou moins développé de l'anachronisme; échos littéraires pour auditeurs cultivés (par exemple, de la Phèdre de Racine dans le Minotaure), voire quiproquos et jeux de mots. Ainsi, dans ce début de dialogue entre les filles de Minos:

PHEDRE. Soeur Ariane, je ne vois rien venir.

ARIANE. Si tu te penches tout le temps, tu finiras par tomber.

Ces pièces ont-elles des qualités proprement dramatiques? On peut en douter, compte tenu de l'extrême rareté des représentations qui en ont été tentées (une ou deux fois, à ma connaissance, pour chacune). L'intelligence, la culture, l'originalité, la beauté du style sont une chose, la vis dramatica en est une autre. Dans les jugements fort acerbes portés par Marguerite Yourcenar sur les pièces "antiques" de Jean Giraudoux, je n'oserais affirmer qu'il n'y ait pas une petite part d'envie... 


\section{Note}

1- Au cours du Colloque Universitaire "Rencontres autour du théâtre de Marguerite Yourcenar", qui s'est tenu à l'Hôtel de la Monnaie, Quai Conti à Paris, on a pu entendre dans la séance du samedi 18 juin 1989 les communications suivantes:

Vassiliki DIKOPOULOU (Athènes), Le Mythe grec, "admirable chèque en blanc" dans le théâtre Yourcenarien; Maurice DELCROIX (Anvers), Dramaturgie du Mystère d'Alceste; Françoise BONALI (Parme), Destin et Liberté dans Electre. ou la Chute des Masques; Rémy POIGNAULT (Tours), D'Ariane et l'Aventurier à Qui n'a pas son Minotaure?

Ces communications seront publiées dans le Bulletin de la Société Internationale d'Etudes Yourcenariennes (Président: M. R. Poignault, 7 rue Couchot, F 72200 LA FLECHE).

Mme V. DIKOPOULOU achève actuellement la préparation d'un Doctorat d'Etat en Sorbonne sur "Marguerite Yourcenar et la Grèce", dirigé par le Pr. M.-F. Guyard.

Du 3 juin au 9 juillet 1989, en liaison avec une exposition "La vie et l'oeuvre de Marguerite Yourcenar", la Compagnie Jean-Louis BIHOREAU a interprété Qui n'a pas son Minotaure? à l'Hôtel de la Monnaie.

\section{Références Bibliographiques}

BRUNEL, P. Electre ou la Chute des Masques de Marguerite Yourcenar. In: Actes du Colloque International M. Yourcenar (Valencia, 1984), Universidad de Valencia, 1986, p. 27-35.

POIGNAULT, R. Le Mystère d'Alceste: rénovation et métamorphose du mythe. In: Il confronto letterario, Suppl. 5, Pavie, 1986, p. 69-80.

POIGNAULT, R. Qui n'a pas son Minotaure? de M. Yourcenar. In: La Mythologie, clé de lecture du Monde classique - Hommage à R. Chevallier, Caesarodunum, XXIbis, 1986, p. 559-80.

YOURCENAR, M. Théâtre, II (Electre ou la Chute des Masques; Le Mystère d'Alceste; Qui n'a pas son Minotaure?). Paris: Gallimard, 1971; édition princeps: Plon, 1954, pour Electre; Plon, 1963, pour les deux autres pièces. 
JOUAN, F. Trois pièces à sujets antiques de Marguerite Yourcenar. Clássica, São Paulo, 4: 215-222, 1991.

RÉSUMÉ: Marguerite Yourcenar s'est inspiré dans ses oeuvres de la Grèce ancienne et de la Grèce moderne. Des deux volumes de son théâtre complet, le second est consacré à ses trois "pièces grecques": Electre ou la Chute des Masques, Le Mystère d'Alceste et Qui n'a pas son Minotaure? - suivies d'études pour chacune de ces pièces, des pages admirables de critique et de style. Ces soeuvres sont marquées par des questions métaphysiques qui sont au coeur de ses oeuvres poétiques ou romanesques et qui tournent autour de l'Homme, de la Liberté et de la Fatalité, de la Personnalité et de l'Inconscient. Malgré l'intelligence, la culture, l'originalité et la beauté du style, ces pièces n'ont pas des qualités proprement dramatiques, d'où la rareté des représentations.

MOTS CLÉS: Marguerite Yourcenar, théatre grec antique, théatre moderne. 